\title{
Insulin Resistance: A Hidden Risk Factor for Gastric Cancer?
}

\author{
Yoon Jin Choi \\ Department of Internal Medicine, Korea University Guro Hospital, Seoul, Korea
}

See “Insulin Resistance Is Associated with Early Gastric Cancer: A Prospective Multicenter Case Control Study" by Hye Jung Kwon, et al. on page 154, Vol. 13. No. 2, 2019

While the incidence of stomach cancer is decreasing, stomach cancer is still the fifth most common cancer and the third leading cause of cancer-related mortality in both sexes worldwide. Globally, 723,000 deaths (8.8\% of the total) related to stomach cancer occurred in 2012. ${ }^{1}$ Apart from several well-known risk factors including Helicobacter pylori infection, smoking, alcohol consumption, family history, and frequent consumption of saltpreserved foods and dietary nitrite, ${ }^{2}$ being overweight or obese is a possible cause of cancers of cardia. ${ }^{3}$ Because diabetes mellitus (DM), as well as a prevalent Westernized lifestyle, has become a global problem, there are numerous studies on the association between DM and cancer. However, epidemiologic studies on the possible associations between DM and gastric cancer are lacking, and the results are inconclusive. Fasting glucose was an independent risk factor for gastric cancer in previous studies, ${ }^{4}$ while most of the studies investing the relationship between diabetes and risk of GC found a null association between the two diseases. ${ }^{5}$

In this issue of Gut and Liver, Kwon et al. ${ }^{6}$ compared fasting glucose and insulin, glycated hemoglobin $(\mathrm{HbA1c})$, homeostasis model assessment of the insulin resistance (HOMA-IR) index, HOMA $\beta$-cell function and lipid profile between 63 patients with early gastric cancer and 63 matched control subjects in South Korea. They have reported that patients with early gastric cancer had a higher level of fasting glucose, fasting insulin, HOMA-IR and total cholesterol compared to the non-gastric cancer control.

While many previous studies have used self-reported diabetes history as a basis for exposure assessment, ${ }^{5}$ this study showed that the insulin resistance is associated with gastric cancer by measuring several objective factors such as fasting insulin and glucose level. Moreover, by excluding users of hypoglycemic agents or lipid-lowering drugs, the present study has the advantage of controlling the confounding effect of drugs. There is increasing evidence to report the beneficial effects of metformin or statins on gastric cancer prevention. ${ }^{7,8}$ Kwon et al. ${ }^{6}$ also focused on the early stage of gastric cancer to reduce the possible reversed causal relationship such as secondary hyperglycemia.

However, there are inevitable limitations of the case-control study including the uncertainty about a temporal relationship. Although authors have stated no significant difference in frequencies of smoking and alcohol consumption, we could not exclude the possibility that unhealthy lifestyle including alcohol consumption or dietary habit, which underlie the gastric carcinogenesis, causes both insulin resistance and gastric cancer in parallel. Similarly, H. pylori infection could be a possible confounder. Two Japanese studies have shown that the impaired fasting blood glucose and high hemoglobin $\mathrm{A} 1 \mathrm{c}^{9,10}$ are associated with an increased risk of gastric cancer, only in $H$. pylori positive subjects. Although Kwon et al. had data about H. pylori seropositivity, it would be difficult to evaluate the true effect of $\mathrm{DM}$ or insulin resistance on the gastric carcinogenesis, due to the relatively small sample size. Despite these limitations, this study of Kwon et al. has an important clinical meaning because authors provided a clue to other modifiable risk factors of gastric cancer besides $H$. pylori, smoking and alcohol consumption.

Given that main hypothesized mechanisms for the carcinogenic effect of diabetes underlies insulin resistance with secondary hyperinsulinemia, the generally null association between a history of diabetes and the risk of gastric cancer in the previous literature is somewhat surprising. Because gastric cancer has been considered more heterogeneous diseases compared to colon cancer, it may be important to discern between the subgroups in investigations of gastric cancers. For the future per-

\footnotetext{
Correspondence to: Yoon Jin Choi (https://orcid.org/0000-0002-1922-9388)

Department of Internal Medicine, Korea University Guro Hospital, 148 Gurodong-ro, Guro-gu, Seoul 08308, Korea

Tel: +82-2-2626-1778, E-mail: erica0007@gmail.com

pISSN 1976-2283 eISSN 2005-1212 https://doi.org/10.5009/gnl19060

(a) This is an Open Access article distributed under the terms of the Creative Commons Attribution Non-Commercial License (http://creativecommons.org/licenses/by-nc/4.0) which permits unrestricted non-commercial use, distribution, and reproduction in any medium, provided the original work is properly cited.
} 
spective, well-designed large prospective epidemiological studies with detailed data about lifestyle and subgroup analyses based on cancer location (i.e., cardia vs non-cardia), histology subtype and gender are required to confirm the association between diabetes (or insulin resistance) and gastric carcinogenesis. Whether patients with impaired glucose control should undergo a more careful endoscopic survey may be the next step.

\section{CONFLICTS OF INTEREST}

No potential conflict of interest relevant to this article was reported.

\section{REFERENCES}

1. Jemal A, Bray F, Center MM, Ferlay J, Ward E, Forman D. Global cancer statistics. CA Cancer J Clin 2011;61:69-90.

2. Rugge M, Fassan M, Graham DY. Epidemiology of gastric cancer. In: Strong V, ed. Gastric Cancer. Cham: Springer, 2015;23-34.

3. Yang $\mathrm{P}$, Zhou $\mathrm{Y}$, Chen $\mathrm{B}$, et al. Overweight, obesity and gastric cancer risk: results from a meta-analysis of cohort studies. Eur J Cancer 2009;45:2867-2873.

4. Tseng $\mathrm{CH}$. Diabetes conveys a higher risk of gastric cancer mortal- ity despite an age-standardised decreasing trend in the general population in Taiwan. Gut 2011;60:774-779.

5. Miao ZF, Xu H, Xu YY, et al. Diabetes mellitus and the risk of gastric cancer: a meta-analysis of cohort studies. Oncotarget 2017;8:44881-44892.

6. Kwon HJ, Park MI, Park SJ, et al. Insulin resistance is associated with early gastric cancer: a prospective multicenter case control study. Gut Liver 2019;13:154-160.

7. Cheung KS, Chan EW, Wong AY, et al. Metformin use and gastric cancer risk in diabetic patients after Helicobacter pylori eradication. J Natl Cancer Inst. Epub 2018 Oct 16. https://doi.org/10.1093/ jnci/djy144.

8. Singh PP, Singh S. Statins are associated with reduced risk of gastric cancer: a systematic review and meta-analysis. Ann Oncol 2013;24:1721-1730.

9. Yamagata H, Kiyohara Y, Nakamura S, et al. Impact of fasting plasma glucose levels on gastric cancer incidence in a general Japanese population: the Hisayama study. Diabetes Care 2005;28:789-794.

10. Ikeda F, Doi Y, Yonemoto K, et al. Hyperglycemia increases risk of gastric cancer posed by Helicobacter pylori infection: a population-based cohort study. Gastroenterology 2009;136:1234-1241. 